\title{
COMMENTARY
}

\section{Defying birth defects through diet?}

Krista S Crider' and Lynn B Bailey*2

\begin{abstract}
The risk of certain birth defects can be modified by maternal diet. A high-fat maternal mouse diet has recently been reported to substantially increase the penetrance of birth defects known to be associated with a deficiency of transcription factor Cited 2 as well as induce cleft palate. These effects were associated with a more than twofold reduction in embryonic expression of Pit $\times 2 c$. This investigation suggests the need to further explore this provocative gene-diet interaction in human studies.
\end{abstract}

\section{Introduction}

The initial formation of many major structures of the human embryo takes place during a very narrow window of time in the first weeks after conception, often before a woman even knows she is pregnant. Any change in the early embryonic environment, including nutrient availability, has the potential to adversely or positively affect the developing embryo. Evidence is evolving that specific components of maternal diet can play a critical role in modifying the risk for birth defects.

The best example of the influence of maternal diet on birth defect risk is the intake of folic acid for prevention of neural tube defects (NTDs) [1]. Randomized controlled folic acid intervention trials demonstrating the effectiveness of folic acid for NTD risk reduction have led to the current public health recommendation, as well as folic acid fortification of the food supply [1]. Prevention of NTDs with supplemental folic acid is likely to involve a complex interaction between folic acid and multiple genetic factors; this is the focus of ongoing investigations [1].

The success of folic acid supplementation in reducing NTDs has led researchers to evaluate the association of other dietary components, such as fat, with a risk for birth defects. Researchers have found that pre-pregnancy diabetes and obesity are associated with an increased risk

*Correspondence: Lynn B Bailey folate@ufl.edu

2Food Science and Human Nutrition Department, PO Box 110370, University of

Florida, Gainesville, FL 32611, USA

Full list of author information is available at the end of the article for numerous birth defects (for example, specific heart defects, NTDs and cleft palate) [2]. A recent metaanalysis revealed that certain aspects of preconception care reduced the risk for some birth defects among women with diabetes: the risk reductions were associated with changes in behavior, such as increased folic acid use or improved glycemic control, or both (depending on the study) [2]. Both diabetes and obesity are related etiologically to high-fat diets, although the mechanisms by which these conditions are or might be teratogenic during pregnancy are unclear. A high-fat western diet has been shown in a retrospective case-control study to be associated with a more than twofold increased risk for cleft lip, with or without cleft palate [3]. The mechanisms by which dietary fat intake might influence the risk for birth defects remain under investigation.

\section{High-fat diet and the risk for birth defects in mice}

One of the strongest risk factors for many birth defects is a family history of the same defects; this reflects both genetic and environmental risks for conditions with complex inheritance patterns, and, as a result, it has been hypothesized that many birth defects have both genetic and environmental influences. A recent paper from Bentham et al. [4] described the interaction of a high-fat maternal diet and the embryonic Cited 2 genotype in increasing the penetrance of left-right patterning birth defects in mouse embryos. The phenotype of mice deficient in Cited 2 varies both within and between mouse strains. This variable penetrance results in left-right patterning defects that include heart defects (septal, outflow tract, and abnormal ventricular topology with sinistral looping) and right atrial and pulmonary isomerisms [5]. The evidence of increased penetrance (but not complete) in left-right patterning defects in certain mouse strains suggests a role for genetic modifiers of the phenotype, as well as a role for environmental influences. Bentham et al. also examined the possible role of the fat content of the maternal diet on the phenotype. They used a diet designed to mimic the high-fat, calorie-dense diet associated with the current obesity epidemic in the USA. It contained lard in place of starch, which has been shown in previous studies to be associated with weight gain and fasting hyperinsulinemia [6]. The experiment showed that the high-fat maternal diet increased the penetrance of left-right patterning defects in mouse 
embryos (60\% of Cited $2-/$ - on the high-fat diet versus $24 \%$ of Cited $2-/-$ on the control diet). The severity of cardiac defects was greater in heterozygous embryos (Cited2+/-) from the high-fat diet group. Additionally, embryos on the high-fat diet exhibited a cleft palate defect not reported previously for Cited2-/.. There were no defects in wild-type embryos on either diet. Further studies revealed that there were greater than twofold decreases in Pitx2c expression in the embryos from the high-fat diet group with the Cited2-/- genotype. Pitx $2 c$ deficiency is known to be associated with right atrial and pulmonary isomerisms [7]. A high-fat diet increases adiposity and glucose intolerance, as well as fasting hyperinsulinemia. However, it is unclear what aspect of the high-fat diet contributes to the increased penetrance and severity of the Cited 2 genotype [6].

\section{How do these results translate to humans?}

The role of Cited2 in malformations in mice is clear; however, among humans, there have been limited numbers of studies to explore the effects of CITED2 mutations. Sperling et al. [8] examined a group of 392 human study participants with sporadic non-syndromic congenital heart disease, and seven new mutations in CITED2 were found among eight of these participants. Additionally, a recent publication by Yang et al. [9] found three new mutations in CITED2 among 120 human study participants with congenital heart defects with a variety of cardiac lesions. Consistent with the variable mouse phenotype, there were no clear groupings of the types of cardiac defects in the human studies associated with the CITED2 mutations, and even the same deletion resulted in different cardiac defects $[8,9]$.

Mouse models can play a critical role in the discovery of disease pathology because they allow control over variables that are uncontrollable among humans. However, results in mice can lead researchers astray, and results need to be confirmed carefully through use of human studies. For example, we know that folic acid food fortification reduces NTD rates among human populations by $19 \%$ to $32 \%$, and that the dietary supplementation of women of childbearing age with $400 \mu \mathrm{g}$ folic acid per day reduces NTD rates up to $85 \%$ among humans [1]. However, there are over 240 mouse models of NTDs, and only a minority of these models implicate folic acid metabolism through mutations in folate pathway genes or have been shown to be responsive to folic acid supplementation [10]. So, it is unlikely that mouse models alone would have led the scientific community to what has become an effective public health intervention.

\section{Future directions}

The presence of CITED2 mutations in more than $2 \%$ of human study participants with congenital heart defects examined to date suggests that CITED2 might have a role in human malformations [8,9]. Interestingly, for some of the same types of defects (for example, heart defects, NTDs, cleft palate and heterotaxia) seen in mice with Cited 2 mutations fed on a high-fat diet, associations with diabetes or obesity, or both, have been suggested in some human studies of birth defects [6,7]. Significant additional work is needed to determine if a similar interaction of genotype and diet is applicable to human embryogenesis. Larger population-based studies are needed to determine the effects of mutations in CITED2 on the burden of specific types and constellations of malformations among humans, and to show whether these mutations have increased penetrance in the presence of high-fat maternal intake.

A better understanding of the interface of geneenvironment interactions has the potential to increase our knowledge of the etiology of birth defects and lead to better targeted prevention programs. Interventions at the maternal-fetal interface are complex because of the environmental interaction with both the maternal and fetal genomes during critically limited windows of time. Before any changes in dietary recommendations can be made, additional large-scale studies are needed to define the 'at-risk' population, followed by randomized controlled trials to determine whether changes in diet can modify the risk and reduce the burden of birth defects.

\section{Abbreviations}

NTD, neural tube defect.

\section{Competing interests}

The authors declare that they have no competing interests. The findings and conclusions in this report are those of the authors and do not necessarily represent the official position of the Centers for Disease Control and Prevention.

\section{Authors' contributions}

KSC and LBB were involved in the drafting of the manuscript and have reviewed and approved the final version.

\section{Authors' information}

KSC is a geneticist at the National Center on Birth Defects and Developmental Disabilities, Centers for Disease Control and Prevention, and LBB is Human Nutrition Professor, Food Science and Human Nutrition Department, University of Florida.

\section{Author details}

'National Center on Birth Defects and Developmental Disabilities, Centers for Disease Control and Prevention, 1600 Clifton Road, MS E-86, Atlanta, GA 30333, USA. ${ }^{2}$ Food Science and Human Nutrition Department, PO Box 110370 , University of Florida, Gainesville, FL 32611, USA.

Published: 9 February 2011

References

1. Berry RJ, Bailey L, Mulinare J, Bower C: Fortification of flour with folic acid. Food Nutr Bull 2010, 31:S22-35.

2. Wahabi HA, Alzeidan RA, Bawazeer GA, Alansari LA, Esmaeil SA: Preconception care for diabetic women for improving maternal and fetal outcomes: a systematic review and meta-analysis. BMC Pregnancy Childbirth 2010, 10:63.

3. Vujkovic M, Ocke MC, van der Spek PJ, Yazdanpanah N, Steegers EA, 
Steegers-Theunissen RP: Maternal Western dietary patterns and the risk of developing a cleft lip with or without a cleft palate. Obstet Gynecol 2007, 110:378-384

4. Bentham J, Michell AC, Lockstone H, Andrew D, Schneider JE, Brown NA Bhattacharya S: Maternal high-fat diet interacts with embryonic Cited2 genotype to reduce Pitx2c expression and enhance penetrance of leftright patterning defects. Hum Mol Genet 2010, 19:3394-3401.

5. Weninger WJ, Lopes Floro K, Bennett MB, Withington SL, Preis JI, Barbera JP, Mohun TJ, Dunwoodie SL: Cited2 is required both for heart morphogenesis and establishment of the left-right axis in mouse development. Development 2005, 132:1337-1348.

6. Fearnside JF, Dumas ME, Rothwell AR, Wilder SP, Cloarec O, Toye A, Blancher C, Holmes E, Tatoud R, Barton RH, Scott J, Nicholson JK, Gauguier D:

Phylometabonomic patterns of adaptation to high fat diet feeding in inbred mice. PLoS One 2008, 3:e1668.

7. Bamforth SD, Braganca J, Farthing CR, Schneider JE, Broadbent C, Michell AC, Clarke K, Neubauer S, Norris D, Brown NA, Anderson RH, Bhattacharya S: Cited2 controls left-right patterning and heart development through a Nodal-Pitx2c pathway. Nat Genet 2004, 36:1 189-1196.
8. Sperling S, Grimm CH, Dunkel I, Mebus S, Sperling HP, Ebner A, Galli R, Lehrach H, Fusch C, Berger F, Hammer S: Identification and functional analysis of CITED2 mutations in patients with congenital heart defects. Hum Mutat 2005, 26:575-582.

9. Yang XF, Wu XY, Li M, Li YG, Dai JT, Bai YH, Tian J: [Mutation analysis of Cited2 in patients with congenital heart disease]. Zhonghua Er Ke Za Zhi 2010, 48:293-296.

10. Harris MJ, Juriloff DM: An update to the list of mouse mutants with neural tube closure defects and advances toward a complete genetic perspective of neural tube closure. Birth Defects Res A Clin Mol Teratol 2010, 88:653-669.

doi:10.1186/gm223

Cite this article as: Crider KS, Bailey LB: Defying birth defects through diet? Genome Medicine 2011, 3:9. 